\title{
Cranial Electrotherapy Stimulation in Patients Suffering from Medically Unexplained Somatic Symptoms: A Case Series
}

\author{
Sagar Karia ${ }^{1}$, Shorouq Motwani ${ }^{2}$, Krishnapriya Murlimanohar ${ }^{3}$, Avinash Desousa $^{4}$, Nilesh Shah ${ }^{5}$
}

\begin{abstract}
Introduction: Patients with medically unexplained somatic symptoms (MUSS) are encountered commonly in clinical practice. These symptoms are often difficult to treat and do not respond well to medication. There have been some studies demonstrating the efficacy of cranial electrotherapy stimulation (CES) in the management of somatic symptoms. Most studies on CES are for the management of anxiety and there is a dearth of literature with regard to the use of CES in MUSS. This case series is aimed at demonstrating the efficacy of CES in the management of MUSS.

Methods: This case series consisted of patients with MUSS that presented to the psychiatry outpatient department of a tertiary general hospital. Patients with MUSS with no other medical cause and not relieved with available medications were included in the case series. Patients were administered CES daily for 10-20 sessions with each session lasting 30 minutes. The efficacy was evaluated by applying Somatic Symptom Scale-8(SSS-8)and the results of all cases were analyzed.

Results: Thirty-five patients ( 26 females and 9 males) between the ages 18-66 years were part of the case series. Majority of them had a diagnosis of major depressive disorder. There was statistically significant improvement in somatic symptoms after CES sessions in majority of the patients at the end of day 20 (29 of 35 patients).

Conclusions: CES is a safe treatment option for MUSS, and further studies in larger samples to establish its efficacy is warranted.

Keywords: CES, Cranial electrotherapy stimulation, Medically unexplained somatic symptoms, Pain.

Indian Journal of Private Psychiatry (2021): 10.5005/jp-journals-10067-0069
\end{abstract}

\section{INTRODUCTION}

Cranial electrotherapy stimulation (CES) is a nonpharmacological treatment approach for the treatment of anxiety, depression, insomnia, and stress. Various medical conditions like headache, lower back pain, toothache, and fibromyalgia with predominantly pain symptoms have been treated with CES with a fair amount of success. ${ }^{1}$ CES is a US-FDA approved drugfree treatment for pain-related conditions. ${ }^{2}$ CES works by the method of transmitting microcurrents in the range between 50 and $0.5 \mathrm{~mA}$ to the cranium from electrodes attached to both earlobes. ${ }^{3}$ Studies of CES in patients over the last three decades in over 30,000 patients have demonstrated its efficacy in the management of chronic pain with depression and more than $50 \%$ of these patients showed a significant improvement in symptoms with minimal side effects. ${ }^{4}$

In clinical practice, patients with medically unexplained somatic symptoms (MUSS) are often encountered. These symptoms are difficult to treat and do not respond to medications. ${ }^{5}$ Majority of the studies on CES have been in patients with anxiety disorders and there is a dearth of Indian literature with regard to the use of CES. ${ }^{6}$ We have used CES in patients with MUSS in our center and this case series has been presented with the aim of evaluating the efficacy of CES in alleviating symptoms of MUSS and also to assess the tolerability and safety of CES in these patients.

\section{Methodology}

This case series was based on cases with MUSS visiting the psychiatry outpatient set up of a tertiary general hospital. Patients who had somatic complaints which no other medical cause explained and which were not relieved with routine medications were part of the case series. The psychiatric diagnosis of patients
${ }^{1-5}$ Department of Psychiatry, Lokmanya Tilak Municipal Medical College, Mumbai, Maharashtra, India

Corresponding Author: Avinash Desousa, Department of Psychiatry, Lokmanya Tilak Municipal Medical College, Mumbai, Maharashtra, India, Phone: +91-22-26460002, e-mail: avinashdes888@gmail.com

How to cite this article: Karia S, Motwani S, Murlimanohar K, et al. Cranial Electrotherapy Stimulation in Patients Suffering from Medically Unexplained Somatic Symptoms: A Case Series. Ind J Priv Psychiatry 2021;15(1):42-44.

Source of support: Nil

Conflict of interest: None

was done based on diagnostic and statistical manual $5 .^{7}$ The patients were explained the basis of CES as a treatment and offered the same as an optional treatment. Their participation and to opt for CES was voluntary. Patients were told that they would be administered CES for 10-20 sessions with each session being 15-30 minutes once a day. The electrodes will be applied on both the earlobes after cleaning the site of application with medical spirit. The machine used was of Balimed Company, Model No. AT-9. ${ }^{8}$ The machine has six levels of impulse range $(0-15 \mathrm{~Hz})$. The impulse was calculated based on the sensations patients felt on earlobes after starting the machine. On subsequent follow-ups, the impulse strength was increased if required. They would have to visit the outpatient department daily for treatment. A gap of maximum 1 day was permitted to maintain efficacy. The efficacy was evaluated by applying Somatic Symptom Scale-8 (SSS-8) ${ }^{9}$ at baseline, day 7 , and day 14 of treatment. The scale used in the study was the SSS-8 which is an abbreviated 8-item version of the Patient Health Questionnaire-15 questionnaire (PHQ-15). ${ }^{10}$ The PHQ-15 
scale assesses the presence and severity of common somatic symptoms and is among the most widely used and validated self-report measures of somatic symptoms burden. Three PHQ-15 items (menstrual problems, sexual problems, and fainting) were not included in the SSS-8 because of low commonalities with other items, low symptom prevalence, and low associations with measures of functioning, quality of life, and healthcare use. A 5-point response option (0-4) for each SSS-8 item and a 7-day time frame was used to mirror the response options and time frame of the patient-reported outcomes measurement. The total score on SSS- 8 ranged from 0 to $32 .{ }^{11}$ For the entire duration of the study, the patients continued consuming medicines that they were already taking prior to the start of CES. No change in medical treatments was made prior to starting CES. The CES was provided free of cost and the patients staying close to the hospital were those that opted for the study. The number of sessions each patient received was based on feedback responses given by the individual patient. As long as they did not find, benefit sessions were continued. The study was a case series and not a randomized trial hence no ethical clearance was needed for the study. However, a written valid informed written consent was taken from every patient and their caregiver prior to the start of CES treatment.

\section{Results}

Our study included 35 patients with age range of 18 to 66 years. The mean age of the patients was $38.43 \pm 13.01$ years. Twentysix $(74.3 \%)$ female patients and $9(25.7 \%)$ male patients were part of the case series. The psychiatric diagnoses of all patients in the case series is mentioned in Table 1. Details of their medications were not discussed as each had different diagnoses and medicines. Details of CES sessions are mentioned in Table 2. All patients received the sessions for 30 minutes per session. The mean scores on SSS- 8 of all 35 patients on baseline was $15.8 \pm 5.61$. The scores on day 7 and day 14 were $10.6 \pm 4.47$ and $6.23 \pm 3.88$, respectively. Repeated measures MANOVA applied to the score showed that the difference between these scores was statistically significant $(p<0.01)$ both at day 7 and day 14 , thereby showing efficacy right during 1st week (Table 3). All 35 patients completed the all sessions and we had no dropouts. None of the patients experienced major side effects. Since the electrodes were applied to the ear lobes many patients (10 of 35) complained of tingling of the earlobes.

Table 1: Diagnostic profile of sample population

\begin{tabular}{lc}
\hline Diagnosis in which patient had MUSS & No. (\%) \\
\hline Alcohol use disorder & $1(2.8 \%)$ \\
Anxiety disorder & $2(5.8 \%)$ \\
Conversion disorder & $4(11.5 \%)$ \\
Illness anxiety disorder & $1(2.8 \%)$ \\
Major depressive disorder & $16(45.7 \%)$ \\
Schizophrenia with conversion disorder & $1(2.8 \%)$ \\
Somatic symptom disorder & $10(28.6 \%)$ \\
\hline
\end{tabular}

\begin{tabular}{lc}
\multicolumn{2}{l}{ Table 2: Number of sessions received by the patients } \\
\hline Sessions received & No. $(\%)$ \\
\hline 10 & $3(8.6 \%)$ \\
11 & $4(11.4 \%)$ \\
12 & $5(14.3 \%)$ \\
13 & $2(5.7 \%)$ \\
14 & $6(17.1 \%)$ \\
15 & $10(28.6 \%)$ \\
16 & $2(5.7 \%)$ \\
17 & $2(5.7 \%)$ \\
18 & $1(2.9 \%)$ \\
\hline
\end{tabular}

Table 3: Comparison of SSS-8 scores by repeated measures of analysis

\begin{tabular}{lccc}
\hline $\begin{array}{l}\text { SSS-8 score } \\
(N=35)\end{array}$ & Mean (SD) & $\begin{array}{c}\text { SSS-8 paired } \\
\text { differences } \\
(N=35)\end{array}$ & Mean $(S D) t(p)$ \\
\hline Baseline & $15.8(5.65)$ & Baseline-Day 7 & $5.2(2.62), 11.73(<0.01)$ \\
Day 7 & $10.6(4.46)$ & Day 7-Day 14 & $4.37(2.27), 11.36(<0.01)$ \\
Day 14 & $6.23(3.88)$ & Baseline-Day 14 & $9.57(4.3), 13.17(<0.01)$ \\
\hline
\end{tabular}

\section{Discussion}

The study revealed that CES as a treatment gave good results in almost $95 \%$ of the patients over 2 weeks. The improvement in MUSS is due to CES as their primary pathology was already under control, only symptom remaining was that of somatic complaints. While many patients improved, the exact mechanism of action of CES in MUSS remains unclear but it seems that the microcurrents activate specific neurons in the brainstem and that these neurocytes demonstrate increased ability to produce neurotransmitters such as serotonin, norepinephrine, and dopamine. These neurotransmitters later restore brain's normal biochemical homeostasis which had disrupted due to stress. ${ }^{11}$ CES has been shown in studies to directly act on the reticular activating system of the brain which has a key role in regulating the activity of brain waves. ${ }^{12-14}$ The relaxed state that ensues induced by CES helps in reducing stress, mood stabilization, and also it regulates awareness and perception of specific pain types. This explains anxiolytic action of CES. ${ }^{15}$

The primary role of the reticular activating system is in the regulation of electrocortical activity and they influence our emotional states. Electrical stimulation of the periaqueductal gray matter has been shown to activate descending inhibitory pathways of pain from the medial brainstem to the dorsal horn of the spinal cord. Cortical inhibition also may be augmented by CES. ${ }^{16}$

Many patients with MUSS experience side effects with psychotropics which often is a reason for noncompliance to treatment. CES has minimal side effects and being machine-based appears to have an additional placebo effect. It can be safely used as an adjunct to pharmacotherapy or as a standalone alternative therapy combined with supportive counseling. ${ }^{17}$

The current study is just a case study and limited to 35 cases. Another limitation of our study being there was no control group and the sample population was not homogeneous. Larger studies 
with controlled states and comparisons of patients with and without pharmacotherapy shall help us understand the efficacy of CES better. Further larger studies in diverse patient populations are warranted to help us establish the place of CES in treatment algorithms for patients with MUSS.

\section{References}

1. Kirsch DL, Smith RB. The use of cranial electrotherapy stimulation in the management of chronic pain: a review. Neurorehabilitation 2000;14(2):85-94. DOI: 10.3233/NRE-2000-14204.

2. Lichtbroun AS, Raicer MM, Smith RB. The treatment of fibromyalgia with cranial electrotherapy stimulation. J Clin Rheumatol 2001;7(2): 72-78. DOI: 10.1097/00124743-200104000-00003.

3. Schroeder MJ, Barr RE. Quantitative analysis of the electroencephalogram during cranial electrotherapy stimulation. Clin Neurophysiol 2001;112(11):2075-2083. DOI: 10.1016/s13882457(01)00657-5.

4. Shealy $\mathrm{CN}$, Thomlinson P. Safe effective nondrug treatment of chronic depression: a review of research on low-voltage cranial electrical stimulation and other adjunctive therapies. Complement Health Pract Rev 2008;13(2):92-99. DOI: 10.1177/1533210108317232.

5. Sharpe M, Carson A. "Unexplained" somatic symptoms, functional syndromes, and somatization: do we need a paradigm shift? Ann Intern Med 2001;134(9):926-930. DOI: 10.7326/0003-4819-134-9_ part_2-200105011-00018.

6. Kar SK, Sarkar S. Neuro-stimulation techniques for the management of anxiety disorders: an update. Clin Psychopharmacol Neurosci 2016;14(4):330-337. DOI: 10.9758/cpn.2016.14.4.330.

7. American Psychiatric Association. DSM 5. Arlington, VA: American Psychiatric Publishing; 2013. p. 70.

8. Available from: https://www.indiamart.com/proddetail/ces-stressrelive-device-20518718297.html.
9. Gierk B, Kohlmann S, Kroenke K, et al. The somatic symptom scale-8 (SSS-8): a brief measure of somatic symptom burden. JAMA Intern Med 2014;174(3):399-407. DOI: 10.1001/jamainternmed.2013. 12179.

10. Toussaint A, Kroenke K, Baye F, et al. Comparing the Patient Health Questionnaire-15 and the Somatic Symptom Scale-8 as measures of somatic symptom burden. J Psychosom Res 2017;101:44-50. DOI: 10.1016/j.jpsychores.2017.08.002.

11. Gierk B, Kohlmann S, Toussaint A, et al. Assessing somatic symptom burden: a psychometric comparison of the patient health questionnaire-15 (phq-15) and the somatic symptom scale- 8 (SSS-8). J Psychosom Res 2015;78(4):352-355. DOI: 10.1016/j. jpsychores.2014.11.006.

12. Taylor DN, Lee CT, Katims JJ. Effects of cranial transcutaneous electrical nerve stimulation in normal subjects at rest and during psychological stress. Acupunct Electrotherapeut Res 1991;16(1-2): 65-74. DOI: 10.3727/036012991816358080.

13. Kirsch DL, Nichols F. Cranial electrotherapy stimulation for treatment of anxiety, depression, and insomnia. Psychiatric Clinics. 2013 Mar 1;36(1):169-176. DOI: 10.1016/j.psc.2013.01.006.

14. Brunyé TT, Patterson JE, Wooten T, Hussey EK. A critical review of cranial electrotherapy stimulation for neuromodulation in clinical and non-clinical samples. Front Human Neurosci 2021;15:12. DOI: 10.3389/fnhum.2021.625321.

15. Barclay TH, Barclay RD. A clinical trial of cranial electrotherapy stimulation for anxiety and comorbid depression. J Affect Disord 2014;164:171-177. DOI: 10.1016/j.jad.2014.04.029.

16. Gordeev SA. Cognitive functions and the state of nonspecific brain systems in panic disorders. Neurosci Behav Physiol 2008;38(7):707714. DOI: 10.1007/s11055-008-9036-z.

17. olde Hartman TC, Lucassen PL, van de Lisdonk EH, Bor $\mathrm{HH}$, van Weel C. Chronic functional somatic symptoms: a single syndrome? British Journal of General Practice. 2004 Dec 1;54(509):922-927. PMID: 15588538. 